\title{
Determining the usefulness of serum hyaluronic acid levels as a predictor of progression of hand osteoarthritis: longitudinal analysis from the Iwaki cohort
}

\section{Tatsuro Saruga ( $\nabla$ suntatsurous@hirosaki-u.ac.jp )}

Hirosaki Daigaku Igakubu Daigakuin Igaku Kenkyuka

\section{Eiji Sasaki}

Hirosaki Daigaku Igakubu Daigakuin Igaku Kenkyuka

Ryo Inoue

Hirosaki Daigaku Igakubu Daigakuin Igaku Kenkyuka

\section{Daisuke Chiba}

Hirosaki Daigaku Igakubu Daigakuin Igaku Kenkyuka

\section{Saiya Ota}

Hirosaki Daigaku Igakubu Daigakuin Igaku Kenkyuka

Hiroki Iwasaki

Hirosaki Daigaku Igakubu Daigakuin Igaku Kenkyuka

\section{Ryoko Uesato}

Hirosaki Daigaku Igakubu Daigakuin Igaku Kenkyuka

\section{Shigeyuki Nakaji}

Hirosaki Daigaku Igakubu Daigakuin Syakailgaku Kenkyuka

\section{Yasuyuki Ishibashi}

Hirosaki Daigaku Igakubu Daigakuin Igaku Kenkyuka

\section{Research article}

Keywords: Serum hyaluronic acid, Biomarkers, Hand osteoarthritis, Kallman score

Posted Date: March 11th, 2020

DOl: https://doi.org/10.21203/rs.3.rs-16851/v1

License: (c) (1) This work is licensed under a Creative Commons Attribution 4.0 International License. Read Full License 
1 Determining the usefulness of serum hyaluronic acid levels as a predictor of

2 progression of hand osteoarthritis: longitudinal analysis from the Iwaki cohort

4 Tatsuro Saruga ${ }^{1}$ Eiji Sasaki ${ }^{1}, R$ yo Inoue ${ }^{2}$, Daisuke Chiba ${ }^{1}$, Seiya Ota ${ }^{1}$, Hiroki Iwasaki ${ }^{1}$,

$5 \quad$ Ryoko Uesato ${ }^{1}$, Shigeyuki Nakaji ${ }^{2}$, and Yasuyuki Ishibashi ${ }^{1}$

6

$7 \quad$ Institutional Affiliations:

$8{ }^{1}$ Department of Orthopedic Surgery, Hirosaki University Graduate School of Medicine,

9 Hirosaki, Japan

$10{ }^{2}$ Department of Social Medicine, Hirosaki University Graduate School of Medicine,

11 Hirosaki, Japan

12

13 Corresponding author:

14 Tatsuro Saruga, MD,

15 Department of Orthopedic Surgery, Hirosaki University Graduate School of Medicine,

165 Zaifu-cho, Hirosaki, Aomori 036-8562, Japan

17 Tel: $+81-172-39-5083$

18 Fax: +81-172-36-3826 
E-mail: $\underline{\text { suntatsurous@gmail.com }}$

\section{Co-author e-mail addresses:}

22 Eiji Sasaki (e_sasaki@hirosaki-u.ac.jp),

23 Ryo Inoue (inoueryo19781228@yahoo.co.jp)

24 Daisuke Chiba (dachiba@hirosaki-u.ac.jp)

25 Seiya Ota (﹎oota1 @ hirosaki-u.ac.jp)

26 Hiroki Iwasaki (hilopom3@ hotmail.co.jp)

27 Ryoko Uesato (ryoko818@ hirosaki-u.ac.jp)

28 Shigeyuki Nakaji (nakaji@hirosaki-u.ac.jp), and

29 Yasuyuki Ishibashi (yasuyuki@hirosaki-u.ac.jp).

\section{Abstract}

32 Background: Hand osteoarthritis (HOA) causes a significant disfunction in patient's daily life. The predicting factor of hand osteoarthritis has been unknown. We aimed to

34 investigate the usefulness of serum hyaluronic acid (sHA) levels in predicting progression of HOA from a 6-year longitudinal epidemiological study.

36 Design: In 2008, a total of 417 participants in the Iwaki cohort were followed over 6 
years. Hand radiographs were taken at baseline and follow-up and scored according to Kellgren-Lawrence grades and Kallman score for 15 joints. Based on the presence of osteoarthritis, participants were classified into HOA and non-HOA groups. Levels of serum hyaluronic acid (sHA) at baseline were determined by ELISA. Spearman's correlation coefficients between levels of sHA, total number of involved joints, and Kallman score were estimated. Factors related to increasing number of involved joints over a period of six years were analyzed by liner regression analysis.

44 Results: The prevalence of hand osteoarthritis was $19.9 \%$ at baseline and the number of 45 joints involved was $3.6 \pm 2.1$. Levels of sHA in the HOA group at baseline were significantly higher than non-HOA group $(\mathrm{p}<0.0001)$, and correlated with the number of involved joints $(\mathrm{r}=0.399, \mathrm{p}<0.0001)$ and Kallman score $(\mathrm{r}=0.540, \mathrm{p}<0.0001)$. Progression rate was $55.4 \%$ and development rate was $19.1 \%$ over six years. In HOA group, the number of involved joints increased by $4.9 \pm 2.3$. Associated factors were age

$50 \quad(\mathrm{p}<0.0001)$ and higher levels of sHA $(\mathrm{p}<0.0001)$ at baseline.

51 Conclusions: Higher levels of sHA correlated with number of involved joints and

52 Kallman score at baseline. In the longitudinal study, Levels of sHA predicted progression of HOA over six years. 
55 Keywords: Serum hyaluronic acid, Biomarkers, Hand osteoarthritis, Kallman score 56

57 


\section{Introduction}

59 Hand osteoarthritis (HOA) is a common disease in the elderly, and the prevalence as

60 reported radiographically sin population-based studies is 29 to $89 \%$ in middle-aged

61 females [1-5]. HOA causes chronic pain and disabilities that lead to serious problems in

62 activities of daily living. It also has a significant impact on socio-economic status.

63 Although early detection of higher risk patients is necessary in order to begin a

64 preventive approach, patients could not recognize the severity of their HOA until it

65 progressed and caused serious pain and disabilities. Also, the natural history of this

66 disease and therapeutic strategy for preventing progression has not been established.

67 While there were several potential problems regarding high prevalence and progressive

68 activity of this disease, radiographs could not detect minute changes at an early stage.

69 Hence, an easier quantitative evaluation of disease activity needs to be established.

70 As the evaluation tool of synovitis, serum biomarkers have attracted attention.

71 Biomarkers are measured from blood and urine, and many substances that specifically

72 reflect the condition of bone, cartilage and synovitis have been reported [6,7].

73 Biomarkers are suggested as a diagnostic tool and severity predictor of Knee OA,

74 possibly as prognostic predictor [8]. Among them, serum hyaluronic acid (sHA) is

strongly related to symptoms and progression of OA since it reflects the state of 
synovitis. It is gaining attention as a biomarker for OA severity and a predictor of OA progression. Regarding finger OA, it was revealed that higher sHA levels were corelated with the number of osteoarthritic joints in a population-based cohort study [9], and progression of joint space narrowing from longitudinal observations focusing on the patients [10]. However, there has been no longitudinal evaluation of the relationship between long-term radiographic changes in HOA and levels of sHA in epidemiological studies. Furthermore, it is unclear whether sHA levels could be a predictor of HOA progression. The aim of this study was to investigate whether sHA levels could reflect the severity and number of involved joint in HOA. Furthermore, we examined the predictive power of sHA levels in determining the progression of HOA in a longitudinal cohort study. We hypothesized that higher levels of sHA at baseline could predict the newly incident number of involved joints over six years

\section{Methods}

91 Subjects were voluntary participants from the Iwaki Health Promotion Project of 2008 and 2014, a community-based program to prevent lifestyle diseases and improve average life expectancy by performing general health checkups and prophylactic 
94 interventions $[11,12]$. It is an annual program that has been performed in the general

95 population living in the Iwaki area of Hirosaki City located in western Aomori

96 prefecture, Japan, since 2005. This cohort study allows evaluation of many kinds of

97 diseases and disorders from various perspectives and research into the risk factors of

98 locomotive disability. All participants provided written informed consent, and the study

99 was conducted with the approval of the ethics committee of the Hirosaki University

100 School of Medicine.

101

102 Subjects

103 A total of 887 volunteers from approximately 12,000 residents participated in this

104 project in 2008. They were recruited via phone calls from public health nurses and an

105 advertisement in the mass media. Those who had renal failure, liver failure, rheumatoid

106 arthritis, malignant tumors and incomplete questionnaires were excluded from the study.

107 Those who did not undergo radiographic examination were also excluded. A total of 724

108 participants (273males and 451females) were enrolled at baseline. Among them, 417

109 participants (145 males, 272 females) were followed up in the Iwaki 2014 cohort. The

110 follow up rate was $57.5 \%$. Height and body weight were measured, and body mass

111 index (BMI) was calculated. 
114 Blood samples were taken from all participants early in the morning for biochemical

115 examination at baseline and follow-up. Blood sampling was performed before breakfast

116 because circulating sHA increases following a meal [13]. The levels of sHA were

117 determined using the Hyaluronan Assay Kit (Seikagaku Corporation, Tokyo, Japan) [9].

118 The change in sHA levels over six years was defined as $\triangle \mathrm{sHA}$.

\section{Radiographic diagnosis}

121 Radiographs were taken for joint evaluation: postero-anterior view of bilateral hands

122 and antero-posterior view of weight-bearing bilateral knees. The following regions were

123 evaluated from each joint group by trained orthopedic surgeons (R.U. and H.I.). The

124 second to fifth distal interphalangeal (DIP), proximal interphalangeal (PIP), thumb

125 interphalangeal (IP) and carpometacarpal (CMC), and scapho-trapezial joints for each

126 hand were graded according to the Kellgren-Lawrence classification (KL) [14].

127 Radiographic OA was defined as KL grade $\geqq 2$. Participants with at least one involved

128 joint at baseline were assigned to the HOA group while those without radiographic

129 HOA were in the non-HOA group. Furthermore, participants with an increasing number 
130 of involved joints over the period of six years, were classified into the Increasing group.

131 Similarly, the presence of knee OA was also evaluated based on the KL scale in both

132 knee radiographs and defined as OA with KL grade 2 or more. Furthermore, the degrees

133 of HOA were also scored according to the Kallman score [15]. Individual hand joints

134 were assessed for the presence of osteophytes (graded 0-3), joint space narrowing (0-3),

135 subchondral sclerosis (0-1), subchondral cysts (0-1), lateral deformity (0-1), and

136 collapse of central joint cortical bone (0-1) with a total of 208 points. To investigate the

137 intra-observer reliability of the scale, 20 randomly selected hand radiographs were

138 scored by the same reader, and two orthopedists (RU and HI) also scored the 20

139 radiographs to assess the inter-observer reliability. The intra- and inter-observer

140 reliability was assessed by the k-statistic, and they were 0.78 and 0.77 , respectively.

\section{$142 \quad$ Statistical analysis}

143 Data input and calculations were performed with SPSS ver. $12.0 \mathrm{~J}$ (SPSS Inc.,

144 Chicago, IL, USA). In the baseline data, Chi square testing was performed between

145 HOA and non-HOA groups to compare gender, knee OA and smoking status. The

146 Mann-Whitney U test was performed to compare age, BMI, and sHA levels at baseline.

147 Spearman's correlation coefficients were estimated among sHA levels, number of 
148 involved joints, and Kallman score at baseline. In the longitudinal analysis over six

149 years, the baseline levels of sHA and $\triangle$ sHA were compared using the Mann-Whitney U

150 test between Increasing and non-Increasing groups. Furthermore, logistic regression

151 analysis was performed with a model, in which the presence of increasing number of

152 involved joints was a dependent variable, while baseline levels of sHA or $\Delta \mathrm{sHA}$, and

153 relevant factors like age, gender, BMI, smoking, and presence of knee OA were

154 independent variables. A receiver operating characteristic (ROC) analysis was

155 performed to determine whether the levels of sHA at baseline could predict the presence

156 of increasing number of involved joints. We calculated the area under the curve (AUC).

157 The optimal cut-off point was the highest Youden index value (sensitivity + specificity

$158-1)$. A p-value below 0.05 was considered to be statistically significant.

160 Results

161 Eighty-two of 417 participants (19.7\%) were classified into the HOA group (Table 1).

162 The HOA group was older $(p<0.0001)$ and had a higher proportion of females. The

163 prevalence of knee OA was higher $(p<0.0001)$, but no significant difference was

164 observed in BMI (Table 1). The prevalence of HOA at baseline was $16.5 \%$ in males and

$16524.6 \%$ in females. Comparing the prevalence of HOA among interphalangeal joints in 
166 all cases, the prevalence in the thumb CM joint, the thumb IP joint, and the DIP joints

167 were high (Fig.1). The mean levels of baseline sHA were $56.5 \pm 30.1(\mathrm{ng} / \mathrm{ml})$ in the

168 Non-HOA group and $107 \pm 79.3(\mathrm{ng} / \mathrm{ml})$ in the HOA group, which was significantly

169 higher than the non-HOA group $(\mathrm{p}<0.0001)$ (Fig.2A). In addition, there was a

170 significant correlation between baseline levels of sHA and the number of involved joints,

171 and the correlation coefficient was 0.399 ( p <0.0001) (Fig.2B). Similarly, there was a

172 significant correlation between baseline levels of sHA and higher baseline Kallman

173 score, with a correlation coefficient of $0.540(\mathrm{p}<0.0001)$ (Fig.3).

174 Over six years of follow up, $16.8 \%$ of males and $24.2 \%$ of females showed an

175 increase in number of HOA joints on X-ray. Eighty-eight $(21.1 \%)$ participants were

176 classified into the Increasing group and 329 (78.9\%) participants were in the

177 non-Increasing group. The mean levels of baseline sHA in the non-Increasing group was

$17859.3 \pm 33.7 \mathrm{ng} / \mathrm{ml}$, and that of the Increasing group was $92.7 \pm 78.2 \mathrm{ng} / \mathrm{ml}(\mathrm{p}<0.0001)$

179 (Fig.4A). In addition, the values of $\triangle \mathrm{sHA}$ in the Increasing group were also

180 significantly higher than those of the non-Increasing group (Fig.4B). Logistic regression

181 analysis showed that the levels of baseline sHA were significantly correlated with the

182 increasing number of involved joints (Table 2). From the ROC curve, the levels of baseline sHA had a high predictive ability $(\mathrm{AUC}=0.708, \mathrm{p}<0.0001)$ for an increase in 
number of involved joints, in which the cut-off level was $46.1 \mathrm{ng} / \mathrm{ml}$ with an odds ratio of 4.79 (Fig. 5).

\section{Discussion}

188 This is the first population-based longitudinal study to examine the relationship 189 between HOA and levels of sHA. From this epidemiological study, it was revealed that 190 levels of sHA were higher in participants with HOA and correlated with the number of 191 involved joints. Furthermore, longitudinal analysis showed that the increasing number 192 of involved joints over six years was associated with the level of baseline sHA which meant that higher sHA levels could predict the increase in number of involved joints in

194 future. Regarding the relationship between sHA and HOA, similar results were obtained in past cross-sectional studies $[10,16]$, but their validity as a predictor in the longitudinal analysis was not sufficiently investigated.

197 HA is a glycosaminoglycan found in many joint tissues, and an important component 198 of articular cartilage and synovium [10]. It is a marker for synovitis and joint 199 inflammation and is influenced by a variety of factors such as food intake, activity 200 levels, and presence of disease $[18,19]$. Therefore, measurement of sHA is performed using blood collected after an overnight fast with less influence of exercise and food. 
202 Serum hyaluronic acid levels have been considered a promising biomarker for diagnosis

203 of OA and the disease burden [20-22]. Higher sHA levels have been associated with

204 higher KL grades of knee and hip joints [6,9,17,23,24]. In HOA, the burden of

205 osteophytes [20] joint space narrowing, and the number of involved joints were all

206 related to sHA levels. Although the statistical significance of sHA in the HOA group

207 was not demonstrated in the CARRIAGE family study where the association between

208 sHA and HOA was reported for the first time [25], Filcova reported a significant

209 association with sHA in erosive HOA compared to non-erosive HOA in HOA

210 patients[10]. In normal joints, functional and metabolic activities of hyaluronic acid

211 depend on its high levels and high molecular weight [26]. During inflammation,

212 reactive free radicals from neutrophils in synovial fluid damage and depolymerize HA

213 and that leads to a reduction in its high molecular weight [27-29]. This contributes to

214 reduction in synovial fluid viscosity and to dispersion of HA fragments and disaccharide

215 monomers into the circulation [30-31]. Soluble pro-inflammatory cytokines including

216 interleukin -1 and tumor necrosis factor- $\alpha$ can also be responsible for the production of

217 HA in synovial fluid [32]. Small HA oligosaccharides in the joint combine with high

218 molecular mass HA and interfere with the normal chondrocyte-matrix interactions

219 [33,34]. They also activate production and activity of matrix metalloproteinases and 
220 nitric oxide synthesis by articular chondrocytes and inflammatory cells $[35,36]$. This

221 process is involved in the pathogenesis of OA and it can be inferred that the increased

222 levels of sHA in HOA patients can reflect synovial inflammation and destruction of OA

223 cartilage. Moreover, Chen demonstrated that increased levels of sHA in HOA patients is

224 associated with hand symptoms [25]. However, there is still a lack of sufficient studies

225 analyzing biomarkers in HOA.

226 In this study, there was a significant correlation between levels of sHA and number of

227 involved joints in HOA. Furthermore, sHA levels showed a strong correlation with

228 Kallman score. It has been reported that there is a significant correlation between

229 radiological HOA severity and finger pain [3], and also that serum cartilage oligomeric

230 matrix protein (sCOMP), a type of synovial biomarker, showed association with

231 decreased hand function [16]. In knee OA and hip OA, the association between

232 radiographic severity and sHA has been shown [18,23]. From this study, the relationship

233 between radiographic severity of HOA and levels of sHA were also suggested.

234 In this study, it is suggested that the number of involved joints tends to increase in

235 patients with high levels of sHA, and the risk increases 4.79 times when the cut-off

236 levels of sHA is $46.1(\mathrm{ng} / \mathrm{ml})$. Filcova reported that a 2-year follow-up study of $88 \mathrm{HOA}$

237 patients who visited the hospital revealed that Kallman score increased two years later 
238 in patients with high levels of sHA [10]. In agreement with this study, we suggest that

239 serum hyaluronic acid levels may be a prognostic factor in HOA. It is considered that

240 the degree of synovitis and cartilage damage may be associated with these correlations.

241 The knee is the largest among weight-bearing joints and has a large volume of cartilage

242 and synovium. Although the individual sizes of finger joints are very small, their

243 number is significant, resulting in large cartilage and synovial volume. Therefore, it

244 seems that association with sHA was also shown in HOA. However, it is important to

245 note that symptoms of HOA do not necessarily coincide with radiographic findings. In

246 daily practice, there are elderly people who live without pain and ADL restrictions, even

247 though their KL grade is high with a significant number of HOA joints, while patients

248 with low KL grades may develop pain and joint swelling and have a great limitation in

249 ADL. Therefore, it is considered necessary to evaluate both symptoms and prognosis

250 when considering the pathology of HOA.

251 This study has several limitations. First, we did not evaluate hand function such as grip

252 strength, handedness, pain and range of motion at the finger joint. Secondly, we did not

253 investigate detailed evaluations of erosion in radiographic images. We assessed joints

254 using anterior-posterior radiographs of the hand. Strictly speaking, it may have been

better to use lateral views to assess OA in the hand joints [37]. However in previous 
256 cohort studies, the anterior-posterior view was used to assess OA in all the hand joints

$257 \quad[1,2,3,5,38,39]$; thus, comparing the prevalence of OA among them might be beneficial.

258 Third, the intake of hyaluronic acid supplements has not been evaluated. Fourth, it is

259 cited that familial OA and OA that co-morbidly affects the whole body other than the

260 knee joint should not be evaluated. Previous studies highlighted the relationships

261 between increased levels of sHA and knee and finger OA but no relationships to other

262 forms of OA [9].

263 Despite these limitations, our results show that the number of involved joints in the

264 hand gradually increased if the baseline levels of sHA was high. In addition, a

265 significant correlation between the number of involved joints and Kallman score to the

266 levels of sHA was also seen, supporting the previous report that sHA plays an important

267 role in the pathogenesis of HOA. This study is the first report from a long-term

268 longitudinal epidemiological study of the general population concerning the relationship

269 between serum hyaluronic acid levels and HOA.

\section{Conclusion}

272 Serum hyaluronic acid levels correlated significantly with the presence of HOA, the number of joints involved and the Kallman score. In the longitudinal study, sHA was 
274 associated with an increase in number of involved joints after six years, suggesting its

275 usefulness as a predictor of HOA progression.

276

277 Abbreviations

278 HOA Hand osteoarthritis

279 sHA Serum hyaluronic acid

280 BMI Body mass index

281 DIP Distal interphalangeal

282 PIP Proximal interpharlangeal

283 IP Interphalangeal

284 CMC Carpometacarpal

285 KL Kellgren-Lawrence classification

286 ROC Receiver operating characteristic

$287 \quad$ AUC Area under the curve

288 sCOMP Serum cartilage oligomeric matrix protein

289

290 Acknowledgements

291 We are extremely grateful to all the participants in the Iwaki Health Promotion Project 
292 and to the staff of our department, who conducted the interviews and collected the data.

293 We would like to thank Editage (http://www.editage.jp) for the English language 294 editing.

295

296 Funding

297 This study was supported in part by a Grant-in-Aid from the Japanese Society for the 298 Promotion of Science (Nos. 21500676, 18K16606, 18K09091), Health Labor Sciences 299 Research Grant, JOA-Subsidized Science Project Research from the Japanese 300 Orthopedic Association, and the Centre of Innovation Program from the Japan Science 301 and Technology Agency (JPMJCE1302). We would like to thank Editage 302 (www.editage.jp) for English language editing.

\section{$304 \quad$ Availability of data and materials}

305 The datasets used and analyzed in the current study are available from the 306 corresponding author on reasonable request.

\section{Author contributions}

309 All authors were involved with the design of the study, interpretation of data, critical 
310 revising of the manuscript and approving the final version for submission. TS, ES and

311 HI were primarily responsible for the data acquisition, and TS primarily did the initial

312 analysis of the data and drafted the manuscript. TS and ES take full responsibility of the

313 integrity of the work from inception to finished article.

\section{Ethics approval and consent to participate}

316 The Ethics Committee of the Hirosaki University Graduate School of Medicine

317 approved the study, and all participants provided written informed consent before 318 participation.

\section{Consent for publication}

321 Consent for publication was not required as no identifying personal information is 322 being published in this manuscript.

\section{Competing interest}

325 The authors declare that they have no competing interests.

\section{References}


1. Dahaghin S, Bierma-Zeinstra SM, Ginai AZ, Pols HA, Hazes JM, Koes BW. Prevalence and pattern of radiographic hand osteoarthritis and association with pain and disability (the Rotterdam study). Ann Rheum Dis. 2005; 64(5): 682-87.

2. Zhang Y, Xu L, Nevitt MC, Niu J, Goggins JP, Aliabadi P, et al. Lower prevalence of

3. Kodama R, Muraki S, Oka H, Lidagka T, Kagotani R, Yoshimura N, et al. Prevalence of hand osteoarthritis and its relationship to hand pain and grip strength in Japan: the third survey of the ROAD study. Modern Rheumatology. 2016; 26(5): $767-73$

4. Dillon CF, Hirsch R, Rasch EK, Gu Q. Symptomatic hand osteoarthritis in the United States: prevalence and functional impairment estimates from the third U.S. National Health and Nutrition Examination Survey, 1991-1994. Am J Phys Med Rehabil. 2007; 86(1): 12-21.

5. Haara MM, Manninen P, Kroger H, Arokoski JP, Karkkainen A, Knekt P, et al. Osteoarthritis of finger joints in Finns aged 30 or over: prevalence, determinants, and association with mortality. Ann Rheum Dis. 2003; 62: 151-8. 

sectional evaluation of biochemical markers of bone, cartilage, and synovial tissue metabolism in patients with knee osteoarthritis: relations with disease activity and joint damage. Ann Rheum Dis. 2001; 60(6): 619-26.

7. Ishijima M, Watari $T$, Naito $K$, Kaneko H, Futami I, Tomonaga A, et al.

8. Golightly YM, Marshall SW, Kraus VB, Renner JB, Villaveces A, Casteel C, et al. hyaluronic acid as a potential marker with a predictive value for further radiographic progression of hand osteoarthritis. Osteoarthritis Cartilage. 2009; 17(12): 1615-19. 

seen on ultrasonography affects the development of radiographic knee osteoarthritis: a 3-year prospective cohort study. Clin Rheumatol. 2017; 36: 2557-64.

13. Engström-Laurent A. Changes in hyaluronan concentration in tissues and body fluids in disease states. CIBA Found Symp. 1989; 143: 233-40

14. Kellgren JH, Lawrence JS. Radiological assessment of osteoarthrosis. Ann Rheum. 1957; 16: 494-502.

15. Kallman DA, Wigley FM, Scott WW Jr, Hochberg MC, Tobin JD. New radiographic grading scales for osteoarthritis of the hand. Reliability for determining prevalence and progression. Arthritis Rheum. 1989; 32(12): 1584-91

16. Aslam I1, Perjar I, Shi XA, Renner JB, Kraus VB, Golightly YM, et al. Associations between biomarkers of joint metabolism, hand osteoarthritis, and hand pain and function: The Johnston County Osteoarthritis Project. J Rheumatol. 2014; 41(5): 938-44. 

biochemical markers serve as surrogates for imaging in knee osteoarthritis? Arthritis Rheum. 2007; 56(12): 4038- 47.

18. Inoue R, Ishibashi Y, Tsuda E, Yamamoto Y, Matsuzaka M, Takahashi I, et al. Knee osteoarthritis, knee joint pain and aging in relation to increasing serum hyaluronan level in the Japanese population. Osteoarthritis Cartilage. 2011; 19(1): 51-7.

19. Fraser JR, Gibson PR. Mechanisms by which food intake elevates circulating levels of hyaluronan in humans. J Intern Med. 2005; 258(5): 460-6.

20. Kraus VB, Kepler TB, Stabler T, Renner J, Jordan J. First qualification study of serum biomarkers as indicators of total body burden of osteoarthritis. Plos one. 2010; 5(3): e9739.

21. Van WE, DeGroot J, Lems WF, Oostveen JC, Lafeber FP. Serum and urinary biochemical markers for knee and hip-osteoarthritis: a systematic review applying the consensus BIPED criteria. Osteoarthritis Cartilage. 2010; 18(5): 605-12 Cartilage. 2006; 14(8): 723- 7. 
hyaluronan levels and radiographic knee and hip osteoarthritis in African Americans and Caucasians in the Johnston County Osteoarthritis Project. Arthritis Rheum. 2005; 52(1): 105-11.

24. Sharif M, George E, Shepstone L, Knudson W, Thonar EJ, Cushnaghan J, et al. Serum hyaluronic acid level as a predictor of disease progression in osteoarthritis of the knee. Arthritis Rheum. 1995; 38(6): 760-7

25. Chen HC, Shah S, Stabler TV, Li YJ, Kraus VB. Biomarkers associated with clinical phenotypes of hand osteoarthritis in a large multigenerational family: the CARRIAGE family study. Osteoarthritis Cartilage. 2008; 16(9): 1054-9.

26. Balazs EA, Watson D, Duff IF, Rosemans S. Hylaluronic acid in synovial fluid.

27. Baker MS, Green SP, Lowther DA. Changes in the viscosity of hyaluronic acid after 461-7.

28. Al-Assaf S, Navaratnam S, Parsons BJ, Phillips GO. Chain scission of hyaluronan by peroxynitrite. Arch Biochem Biophys. 2003; 411(1): 73-82. 
and synovial fluid in arthritis. Int J Tissue React. 1990; 12(2): 81-9.

419

30. Belcher C, Yaqub R, Fawthrop F, Bayliss M, Doherty M. Synovial fluid chondroitin and keratan sulphate epitopes, glycosaminoglycans, and hyaluronan in arthritic and normal knees. Ann Rheum Dis. 1997; 56(5): 299-307.

31. Dahl LB, Dahl IM, Engström-Laurent A, Granath K. Concentration and molecular weight of sodium hyaluronate in synovial fluid from patients with rheumatoid arthritis and other arthropathies. Ann Rheum Dis. 1985; 44(12): 817-22.

32. Nishida Y, D'Souza AL, Thonar EJ, Knudson W. Stimulation of hyaluronan metabolism by interleukin-1alpha in human articular cartilage. Arthritis Rheum. 2000; 43(6): 1315-26.

33. Maleski MP, Knudson CB. Hyaluronan-mediated aggregation of limb bud mesenchyme and mesenchymal condensation during chondrogenesis. Exp Cell Res. 1996; 225(1): 55-66.

34. Ohno S, Im HJ, Knudson CB, Knudson W. Hyaluronan oligosaccharide-induced activation of transcription factors in bovine articular chondrocytes. Arthritis Rheum. 2005; 52(3): 800-9.

35. Iacob S, Knudson CB. Hyaluronan fragments activate nitric oxide synthase and the production of nitric oxide by articular chondrocytes. Int J Biochem Cell Biol. 2006; 

38(1): 123-33.

437

438

439

440

441

442

443

444

445

446

447

448

449

450

451

452

453

36. Knudson W, Casey B, Nishida Y, Eger W, Kuettner KE, Knudson CB. Hyaluronan oligosaccharides perturb cartilage matrix homeostasis and induce chondrocytic chondrolysis. Arthritis Rheum. 2000;43(5): 1165-74.

37. Eaton RG, Lane LB, Littler JW, Keyser JJ. Ligament reconstruction for the painful thumb carpometacarpal joint: a long-term assessment. J Hand Surg Am. 1984; 9: 692-9.

38. Yoshida S, Aoyagi K, Felson DT, Aliabadi P, Shindo H, Takemoto T. Comparison of the prevalence of radiographic osteoarthritis of the knee and hand between Japan and the United States. J Rheumatol. 2002; 29: 1454-8.

39. Bernard TE, Wilder FV, Aluoch M, Leaverton PE. Job-related osteoarthritis of the knee, foot, hand, and cervical spine. J Occup Environ Med. 2010; 52: 33-8.

\section{Figure titles and legends}

Fig. 1. Prevalence (\%) of hand osteoarthritis on radiography in each joint in men (M, left) and women (W, right).

Fig. 2. Increased levels of sHA in HOA groups compared with non-HOA groups (A) 
454 and correlation of the levels of sHA with the number of involved joints by Spearman's

455 correlation coefficients (B) at baseline.

456

457 Fig. 3. Correlation of levels of sHA with Kallman score in HOA groups by spearman's 458 correlation coefficients at baseline.

459

460 Fig. 4. Increased levels of sHA (A) and $\triangle \mathrm{sHA}(\mathrm{B})$ in Increasing groups compared 461 with non-Increasing groups.

462

463 Fig. 5. The predictability of increasing the number of involved joints by levels of sHA 464 in the receiver operating characteristic curve.

465 
Figures

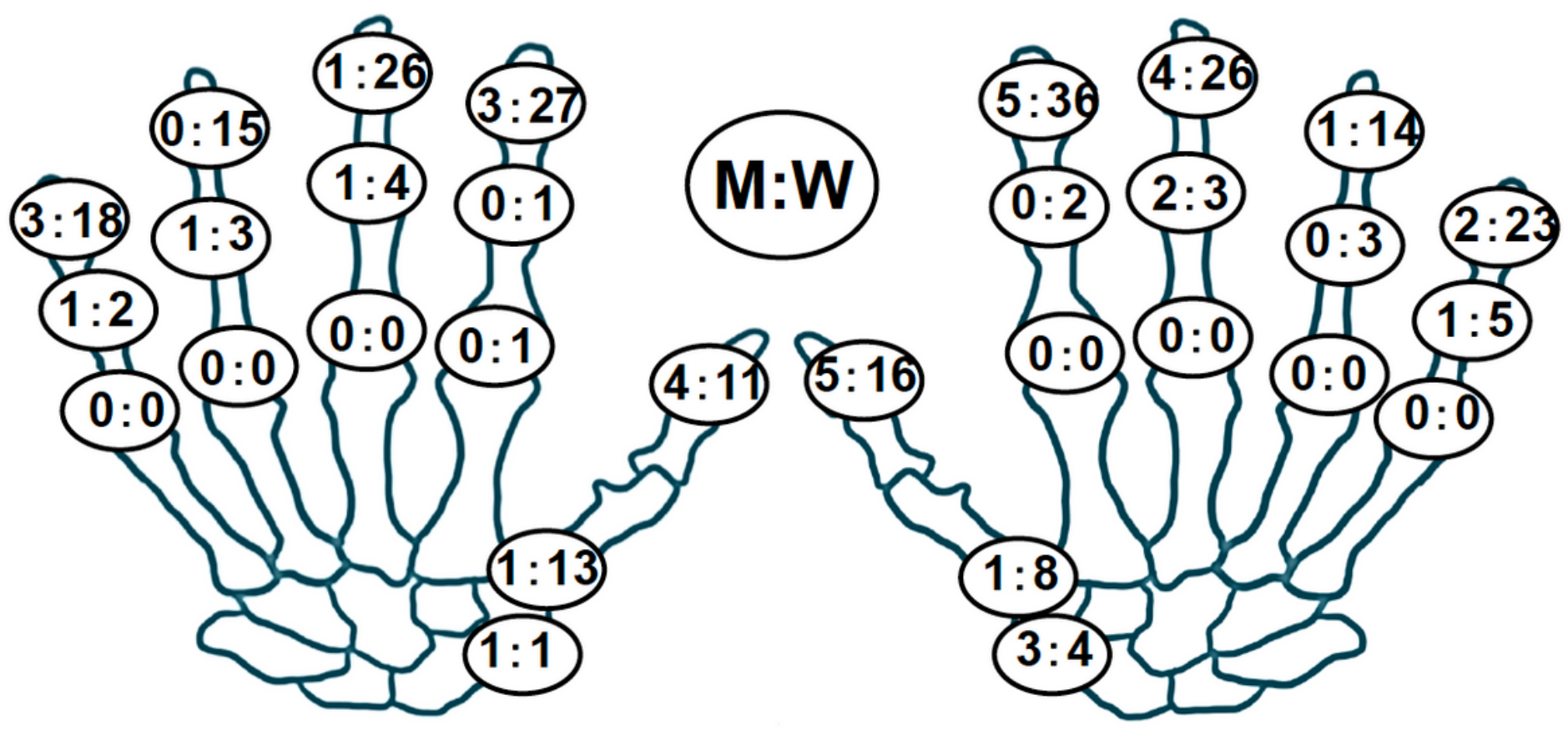

Figure 1

Fig. 1. Prevalence (\%) of hand osteoarthritis on radiography in each joint in men (M, left) and $w$ omen (W, right). 

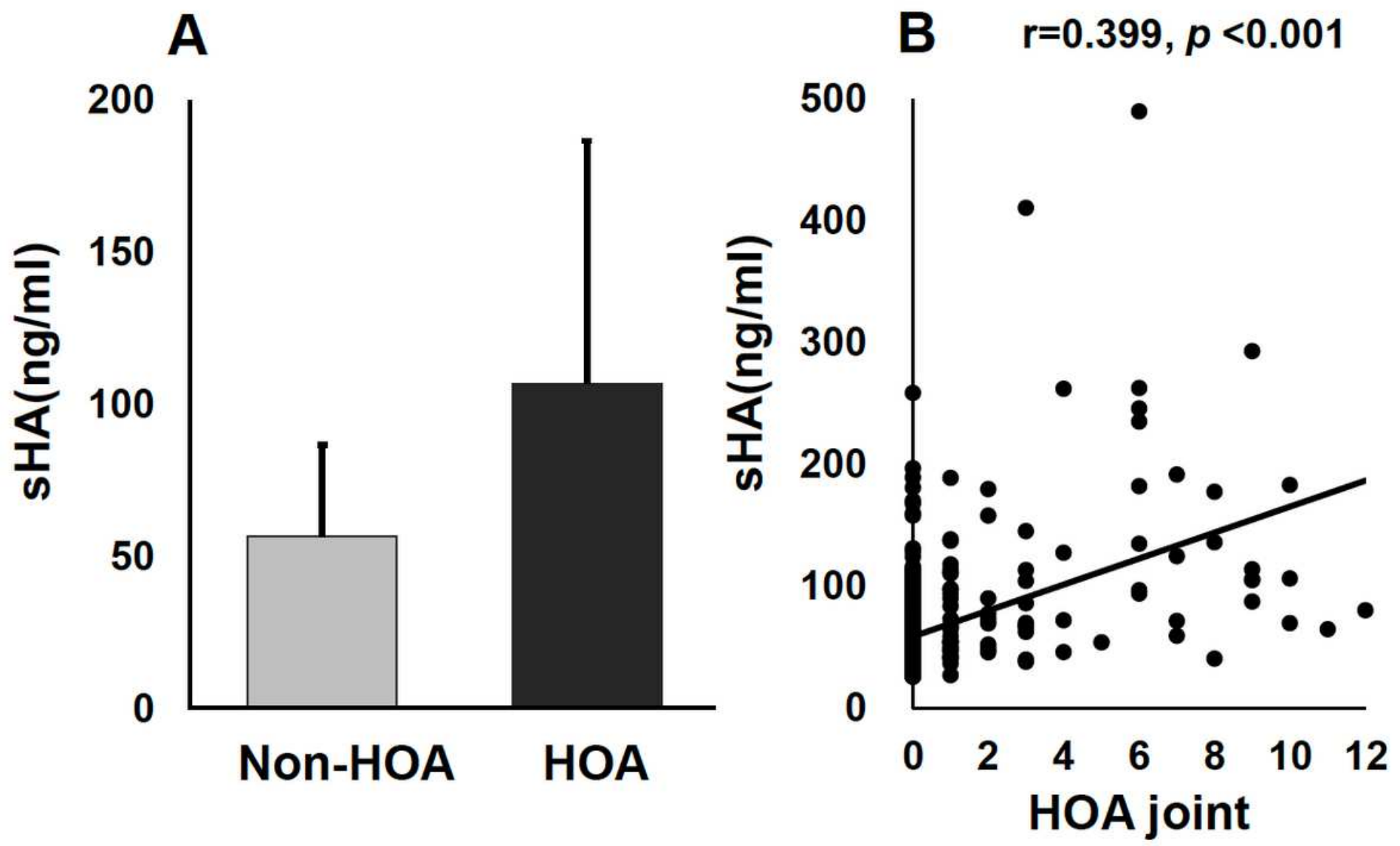

Figure 2

Fig. 2. Increased levels of sHA in HOA groups compared with non-HOA groups (A) and correlation and correlation of the levels of sHA with the number of involved joints by $n$ of the levels of sHA with the number of involved joints by Spearman's correlation coefficients (B) at baseline. 


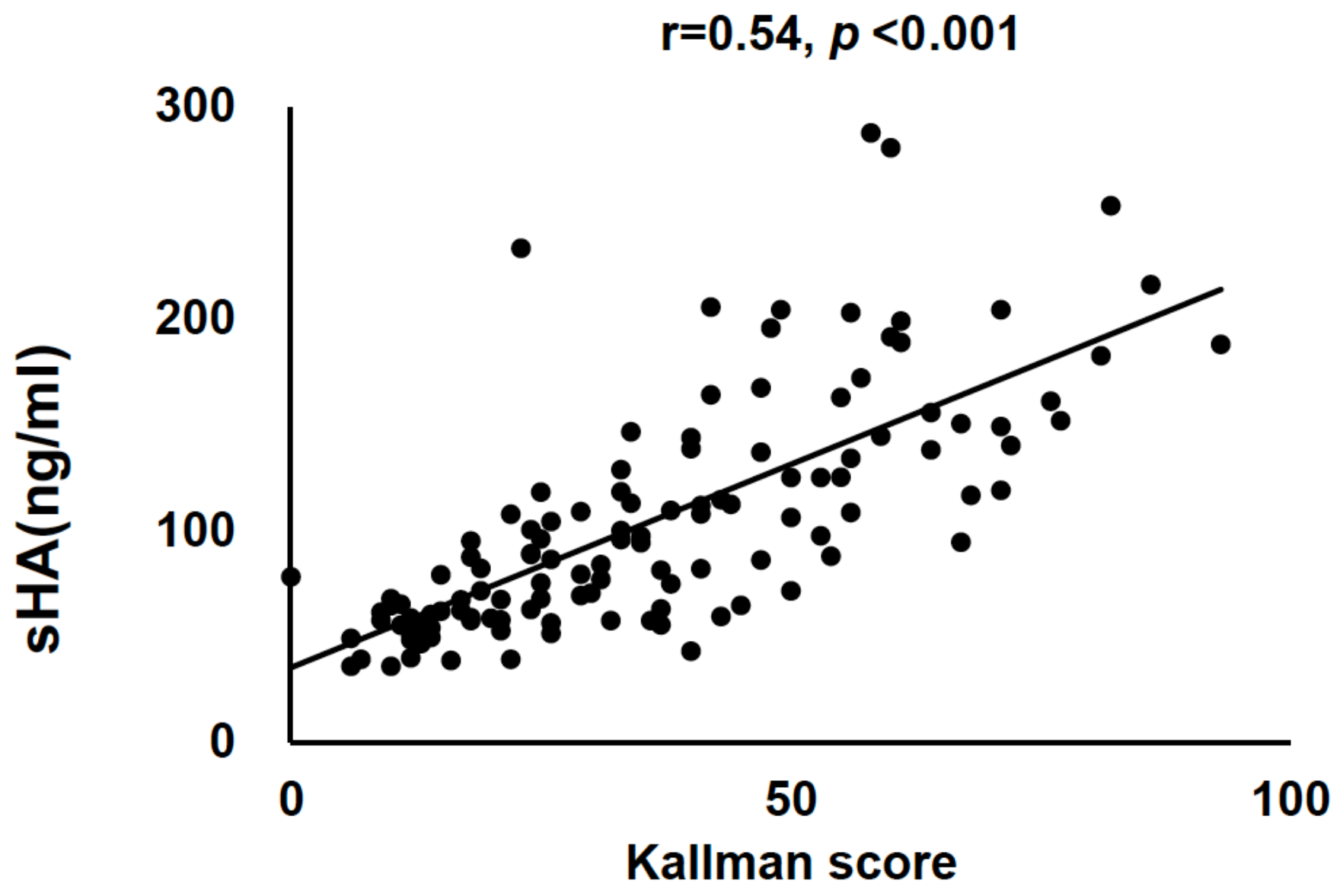

Figure 3

Fig. 3. Correlation of levels of sHA with Kallman score in HOA groups by spearman's correlation coefficients at baseline . 

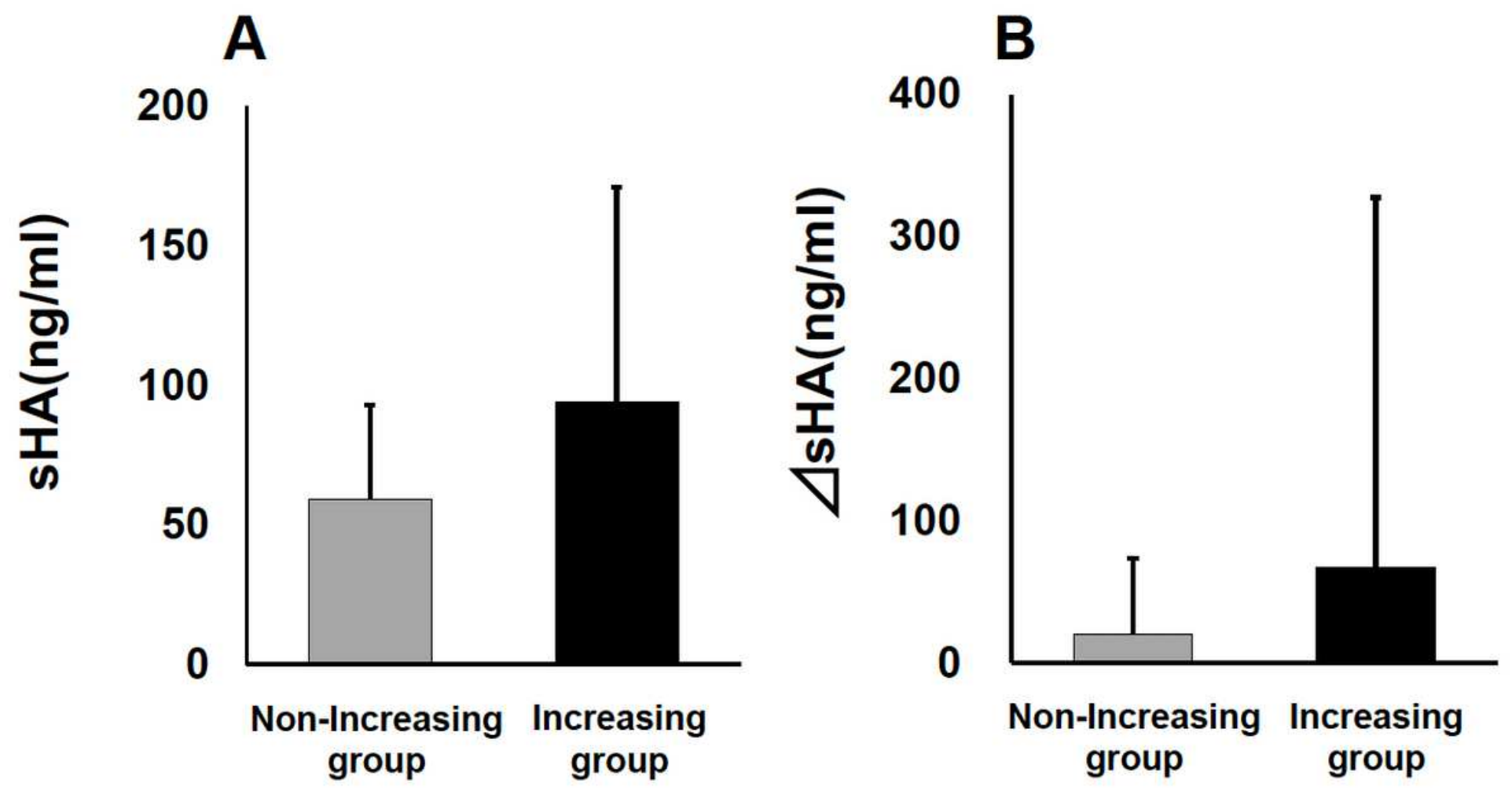

Figure 4

Fig. 4. Increased levels of sHA (A) and sHA (B) in Increasing groups compared with non Increasing groups. 


\section{$\mathrm{AUC}=0.708, \mathrm{sHA}=46.1, \mathrm{Odds}=4.79$}

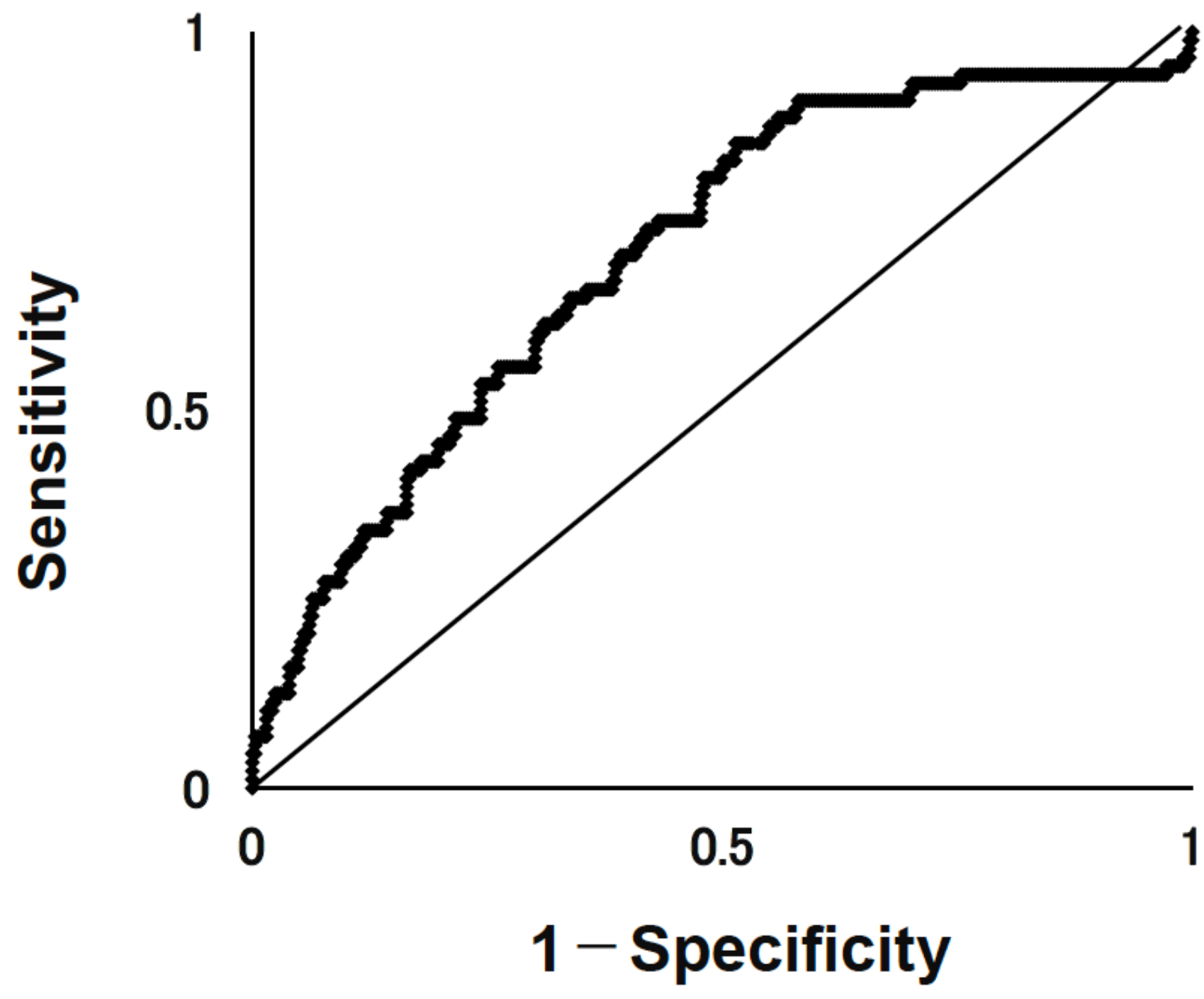

Figure 5

Fig. 5. The predictability of increasing the number of involved joints by levels of sHA in the receiver operating characteristic curve. 